\title{
DLTS investigations on CIGS solar cells from an inline co-evaporation system with RbF post-deposition treatment
}

\author{
Tim Helder $^{1,2, *}$ (D), Ana Kanevce ${ }^{1}$, Andreas Bauer ${ }^{1}$, Mario Zinßer ${ }^{1,2}$ (D), Stefan Paetel ${ }^{1}$ (D), \\ Theresa Magorian Friedlmeier ${ }^{1}$ (D), and Michael Powalla ${ }^{1,2}$ \\ ${ }^{1}$ Zentrum für Sonnenenergie- und Wasserstoffforschung Baden-Württemberg (ZSW), Meitnerstraße 1, 70563 Stuttgart, Germany
${ }^{2}$ Light Technology Institute (LTI), Karlsruhe Institute of Technology (KIT), Engesserstraße 13, 76131 Karlsruhe, Germany
}

Received: 30 June 2021 / Received in final form: 16 December 2021 / Accepted: 3 February 2022

\begin{abstract}
In this study, Deep Level Transient Spectroscopy (DLTS) measurements have been performed on Cu $(\mathrm{In}, \mathrm{Ga}) \mathrm{Se}_{2}$ (CIGS) solar cells from an inline co-evaporation system. The focus of this investigation is directed on the effect of rubidium-fluoride (RbF)-post-deposition treatment (PDT) on the defects in the CIGS absorber layer. Different traps can be identified and their properties are calculated. Herein, different methods of evaluations have been used to verify the results. Specifically, one minority trap around $400 \mathrm{meV}$ was found to show a significant reduction of the trap density due to the alkali treatment. In contrast, a majority trap at approximately $600 \mathrm{meV}$ is unaffected.
\end{abstract}

Keywords: CIGS solar cells / RbF-PDT / defects / DLTS / passivation

\section{Introduction}

Despite the high efficiencies exceeding $23 \%$ achieved with CIGS solar cells in the last years, additional reduction of the non-radiative Shockley-Read-Hall (SRH) recombination through defect passivation is expected to lead to further improvements [1]. A detailed knowledge of defect properties is necessary to identify the dominant loss mechanisms. Herein, DLTS measurements represent a powerful technique for the exploration of the characteristic parameters of these defects [2]. In this study we show how the state-of-the-art modifications of RbF-PDT of the CIGS absorber influences the defects identified by DLTS measurements and the properties of the most active defects.

The PDT of CIGS with alkali elements is a widely-used technique to improve doping and passivate defects and was used in all of the latest CIGS record cells [1,3-5]. It is still an ongoing debate how and where within the solar cell device PDT processes have an effect on defects [6]. Thus, a detailed look on the effect of alkali elements is necessary. On the way to large area devices, modules and mass production, detailed knowledge about the absorber from inline co-evaporation processes and the inherent defects is essential for further development. Thus, this study is based on samples from two similar deposition series from a production-like co-evaporation CIGS system, denoted as "Series I" and "Series II". Within each series, samples with and without PDT have been

\footnotetext{
* e-mail: tim.helder@zsw-bw.de
}

prepared, based on the same CIGS deposition. For detailed information on sample fabrication and preparation, see Section 2. As generally known, the alkali element treatment of these samples improves the open circuit voltage $\left(V_{\mathrm{OC}}\right)$ and the fill factor ( $\mathrm{FF}$ ) which could be linked to the passivation of defects and the increase of effective doping. For Series I, an improvement of $V_{\mathrm{OC}}$ of $7 \mathrm{mV}$ from $709 \mathrm{mV}$ to $716 \mathrm{mV}$ and an increase in $\mathrm{FF}$ of $2 \%$ absolute is observed due to PDT. The $V_{\mathrm{OC}}$ values of the samples from Series II show an increase of $23 \mathrm{mV}$, while the FF remains unchanged. For both series an efficiency gain of 0.2 and $0.3 \%$ is obtained, respectively. The aim of this study is therefore, to examine possible defects influencing $V_{\mathrm{OC}}$ and $\mathrm{FF}$ of the solar cell devices. The second aim is to investigate a possible passivation effect of these defects by PDT and the effect on the cell parameters.

\section{Methods}

\subsection{Sample preparation}

The preparation of CIGS solar cells follows a standard procedure: $2 \mu \mathrm{m}$ CIGS is deposited on molybdenum (Mo) coated soda-lime glass substrates in an inline co-evaporating system by a three-stage process with optional RbF-PDT. The integral $\mathrm{Ga} /(\mathrm{Ga}+\mathrm{In})(\mathrm{GGI})$ ratio ranged between 0.27 and 0.29 . After a rinsing step, $50 \mathrm{~nm}$ cadmium sulfide $(\mathrm{CdS})$ as a buffer layer is deposited by a thiourea-based chemical bath deposition (CBD) recipe [7]. A $90 \mathrm{~nm}$ thick high-resistive layer ( $\mathrm{HRL}$ ) of intrinsic zinc oxide (i-ZnO) is prepared on top by radio frequency (RF) magnetron sputtering, followed by a 
$250 \mathrm{~nm}$ layer of direct current (DC) sputtered aluminum doped zinc oxide $(\mathrm{ZAO})$ as transparent conductive oxide (TCO). Finally, a $\mathrm{Ni} / \mathrm{Al} / \mathrm{Ni}$ metallization grid of approx. $2.5 \mu \mathrm{m}$ is deposited by electron-beam evaporation as electron contact. When comparing samples with and without PDT, both samples have been prepared within the same processes and the only difference is the PDT step. This procedure is described in more detail in literature [8].

For DLTS measurements, the active area of the selected cells have been reduced in order to decrease the capacitance and thus to enable the measurements. The measurement bridge of the DLTS system is the limiting factor, so an area of $1 \mathrm{~mm}^{2}$ was defined around the metallization pad by mechanical scribing.

\subsection{DLTS measurement and evaluation techniques}

DLTS measurements have been conducted with a PhysTech Hera DLTS system. If not stated otherwise, a reverse voltage of $U_{R}=-2 \mathrm{~V}$ and a pulse voltage of $U_{P}=0 \mathrm{~V}$ was used. As pulse width $\left(t_{\mathrm{p}}\right)$ two different lengths of $1 \mathrm{~ms}$ and $50 \mathrm{~ms}$ have been applied. For the standard scan, the values of time window $\left(t_{\mathrm{w}}\right)$ were chosen between 1 and $100 \mathrm{~ms}$. The measurements were carried out in dark and in the temperature range between 80 and $330 \mathrm{~K}$. Due to insufficient thermal conduction of the glass substrate and the non-perfect thermal bonding to the stage, the sample temperature can differ up to $10 \mathrm{~K}$ from the stage temperature and thus reduce the accessible measurement range. All plots of DLTS signals or temperature scans are showing the b1 signal which represents a calculation of $\Delta C$ of the capacitance transients with a sine as weighting function and plotted over the sample temperature. By default the used DLTS system from PhysTech defines the sign of the DLTS peaks as follows: majority traps occur as a maximum in the temperature scan, and minorities as a minimum. The extrema in temperature scans have been evaluated, resulting in a data set in the Arrhenius plot for each trap, with the emission time constant $\tau$ or the emission rate $e_{\mathrm{T}}$ plotted over the inverse temperature $1000 / T$. From a linear regression of these data, the trap properties energetic position $\left(E_{\mathrm{T}}\right)$ and capture cross-section $\left(\sigma_{\mathrm{c}}\right)$ can be extracted following the emission equation:

$$
e_{\mathrm{T}}=N_{\mathrm{C}, \mathrm{V}} \sigma_{\mathrm{C}} v_{\mathrm{th}} \exp \left(-E_{\mathrm{T}} / k T\right)
$$

where $N_{\mathrm{C}, \mathrm{V}}$ is the density of states of electrons and holes, respectively, $v_{\text {th }}$ is the thermal velocity, $k$ is the Boltzmann constant and $T$ is the temperature. In this study all energetic positions are given with respect to the valence band edge $E_{\mathrm{V}}$, due to the p-doping of the investigated absorber layer. In order to calculate the trap density $\left(N_{\mathrm{T}}\right)$, the amplitude of the recorded capacitance transients $\Delta C$, the capacitance of the sample at reverse bias $C_{\mathrm{R}}$ and the charge density $N_{\mathrm{S}}$ of the sample are taken into account [9]:

$$
N_{\mathrm{T}}=2 N_{\mathrm{S}} \frac{\Delta C}{C_{\mathrm{R}}} \frac{W_{\mathrm{R}}^{2}}{L_{\mathrm{R}}^{2}-L_{\mathrm{P}}^{2}} .
$$

Here $W_{\mathrm{R}}$ is the space charge region width at reverse bias and $L_{\mathrm{R}}$ and $L_{\mathrm{P}}$ are the positions of the intersections of the trap level and Fermi level at reverse bias and pulse voltage, respectively.

Additionally, we used a second evaluation method called Deep Level Transient Fourier Spectroscopy (DLTFS). This technique samples the measured capacitance transients and forms discrete Fourier coefficients, from a numerical Fourier transformation. From these coefficients the time constant $\tau$ and amplitude for each transient can be calculated immediately. Detailed information to the theory of DLTFS can be found in literature [10].

For reasons of clarity, only the average of the determined trap properties, calculated with different methods, is used in the text and table. If resulting errors are given for trap level properties, the value of the standard deviation is used. Since the evaluation of capture crosssections via Arrhenius plots is strongly error-prone and depends on assumed semiconductor properties, values can vary over orders of magnitude. Hence, no deviation is given for this quantity and only representative values are shown.

For the calculation of effective doping capacitancevoltage (CV) measurements were conducted with the PhysTech DLTS system as well as by measuring at least from -1 to $0.5 \mathrm{~V}$ and performing a linear fit on the undisturbed part of a Mott-Schottky plot. The measurements were conducted at room temperature, in vacuum and under dark conditions in the cryostat.

\section{Results}

For the major part of our samples without RbF-PDT, a significant negative peak around $220 \mathrm{~K}$ can be observed which is dominating the temperature scan for temperatures below $250 \mathrm{~K}$. Figure 1 shows this peak and its movement to lower temperatures with increasing $t_{\mathrm{w}}$ and accordingly smaller rate windows. Due to the sign of the peak, a minority trap can be assumed and therefore this trap is denoted as "E1" in this study. The evaluation results of this minimum indicate an energetic position of $E_{\mathrm{T}}=(425 \pm 25) \mathrm{meV}$, which is approximately $150 \mathrm{meV}$ away from the middle of the band gap of the CIGS absorber.

For a comparable sample with PDT (from the same CIGS deposition) a similar signal can be detected around $200 \mathrm{~K}$. Figure 2 compares DLTS temperature scans of CIGS samples (a) without RbF-PDT treatment and (b) with PDT. Solid lines represent measurements with long filling pulses of $50 \mathrm{~ms}$ and dashed lines represent measurements with short filling pulses of $1 \mathrm{~ms}$. Herein the long pulse measurement of (a) can be compared with the $t_{\mathrm{w}}=10 \mathrm{~ms}$ measurement from Figure 1 . Despite the disturbed peak shape, the corresponding energy level was calculated to $(418 \pm 22) \mathrm{meV}$ and therefore within the error of the without PDT measurement. The important difference of these two signals is the amplitude of these peaks differing from each other by one order of magnitude and thus indicate a strong reduction of emission processes. This reduction can be directly linked with the extracted trap properties of this measurement, listed in Table 1 in row "Series I". With a trap density of $(6 \pm 3) \times 10^{13} \mathrm{~cm}^{-3}$ for the sample without PDT and $(1.6 \pm 0.5) \times 10^{13} \mathrm{~cm}^{-3}$ for the sample with PDT a reduction in trap density by a factor of 3.8 can be evaluated for the E1 trap. This result could indicate 


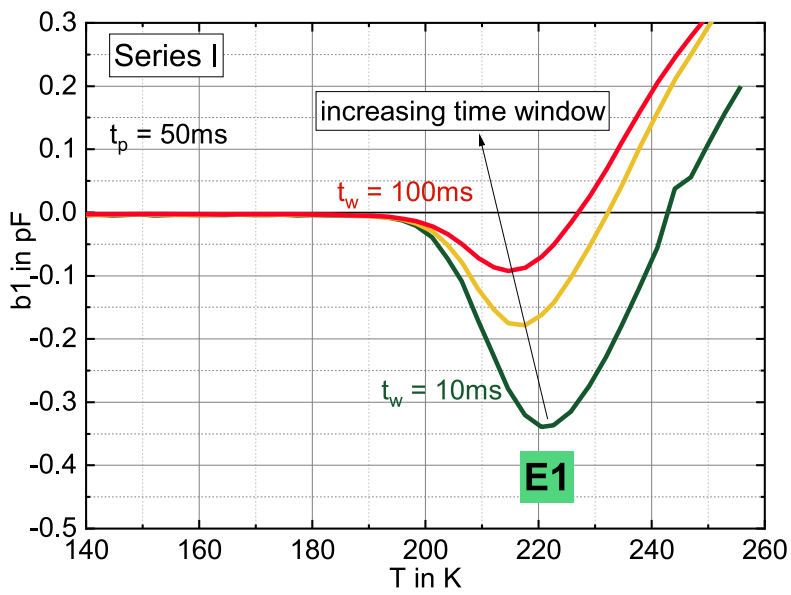

Fig. 1. DLTS temperature scan of a CIGS solar cell without PDT from Series I for different $t_{\mathrm{w}}$ between 10 and $100 \mathrm{~ms}$ and a $t_{\mathrm{p}}$ of $50 \mathrm{~ms}$. The significant minimum at $220 \mathrm{~K}$ is a typical feature of the cells from the used inline evaporation system and can be assigned to a minority trap (E1).
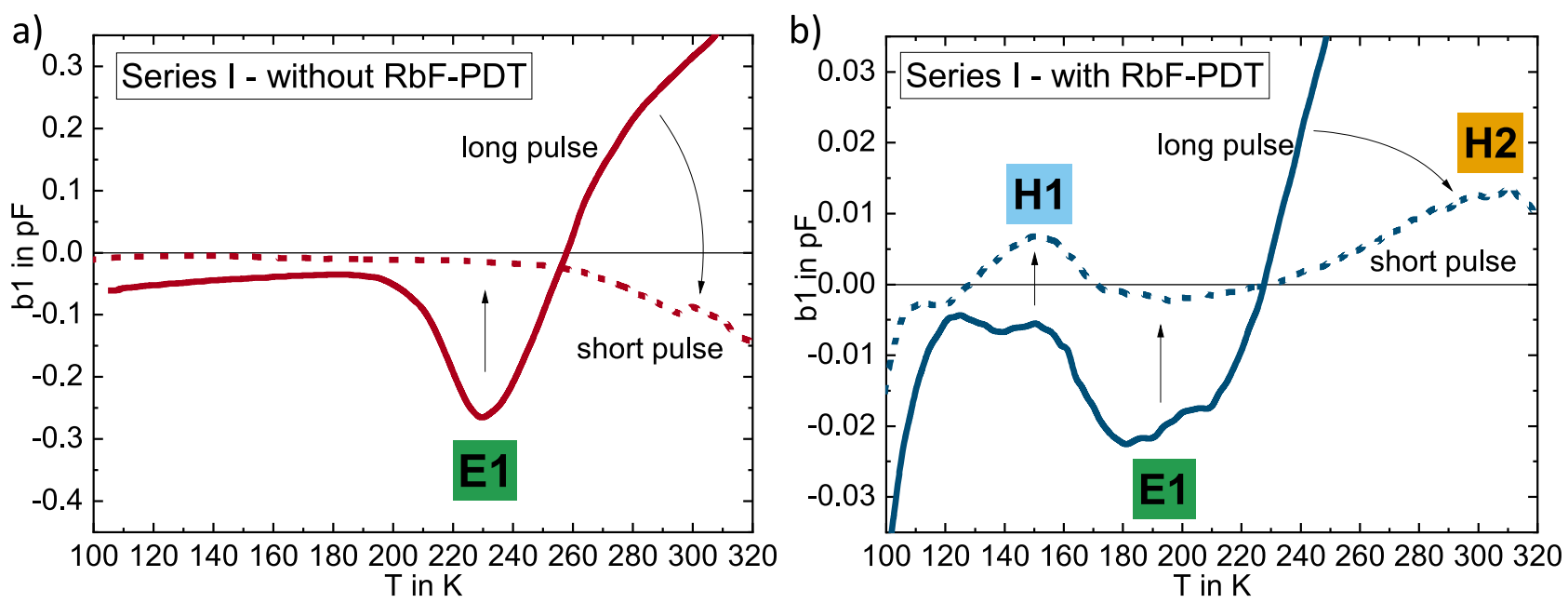

Fig. 2. Comparison of DLTS measurements on CIGS samples from Series I (a) without and (b) with PDT. Solid lines represent measurements with $50 \mathrm{~ms}$ filling pulse, dashed lines with $1 \mathrm{~ms}$. Different pulse widths enable the observation of different traps. For $t_{\mathrm{w}}$ a value of $10 \mathrm{~ms}$ was used.

Table 1. Summary of the calculated trap properties for both investigated deposition series and for samples with and without PDT. In all cases, different evaluation methods have been used to determine the trap properties (manual maximum analysis and DLTFS evaluations). Therefore an average and standard deviation is given for each variation and each trap.

\begin{tabular}{|c|c|c|c|c|c|c|c|}
\hline & Treatment & $\begin{array}{l}\text { Doping } \\
\mathrm{cm}^{-3}\end{array}$ & $\begin{array}{l}V_{\text {oc }} \\
\mathrm{mV}\end{array}$ & Type & $\begin{array}{l}\text { Energy } E_{\mathrm{V}}-E_{\mathrm{T}} \\
\text { meV }\end{array}$ & $\begin{array}{l}\sigma_{\mathrm{n}, \mathrm{p}} \\
\mathrm{cm}^{2}\end{array}$ & $\begin{array}{l}\text { Trap Density } N_{\mathrm{T}} \\
\mathrm{cm}^{-3}\end{array}$ \\
\hline \multirow[t]{3}{*}{ Series I } & w/o PDT & $4.5 \times 10^{15}$ & 709 & E1 & $425 \pm 25$ & $1.2 \times 10^{-15}$ & $(6.0 \pm 3.0) \times 10^{13}$ \\
\hline & $\mathrm{w} / \mathrm{PDT}$ & $8.3 \times 10^{15}$ & 716 & E1 & $418 \pm 22$ & $4.2 \times 10^{-12}$ & $(1.6 \pm 0.5) \times 10^{13}$ \\
\hline & & & & H1 & $198 \pm 22$ & $1.4 \times 10^{-17}$ & $(1.2 \pm 0.3) \times 10^{13}$ \\
\hline \multirow[t]{4}{*}{ Series II } & $\mathrm{w} / \mathrm{o} \mathrm{PDT}$ & $1.4 \times 10^{16}$ & 687 & E1 & $451 \pm 32$ & $1.5 \times 10^{-14}$ & $(2.3 \pm 0.3) \times 10^{13}$ \\
\hline & & & & $\mathrm{H} 2$ & $608 \pm 8$ & $1.2 \times 10^{-15}$ & $(2.5 \pm 1.5) \times 10^{13}$ \\
\hline & $\mathrm{w} / \mathrm{PDT}$ & $2.3 \times 10^{16}$ & 710 & E1 & $459 \pm 11$ & $4.7 \times 10^{-13}$ & $(7.8 \pm 2.3) \times 10^{12}$ \\
\hline & & & & $\mathrm{H} 2$ & $641 \pm 16$ & $2.1 \times 10^{-14}$ & $(3.1 \pm 0.9) \times 10^{13}$ \\
\hline
\end{tabular}




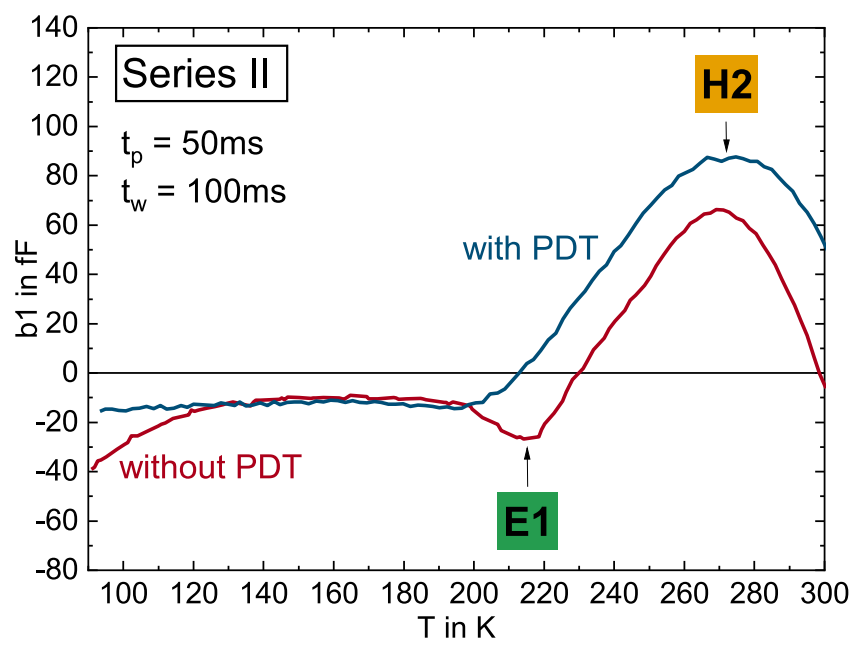

Fig. 3. DLTS signal of a second deposition series, with and without PDT. A minority trap E1 $(\approx 400 \mathrm{meV})$ and a majority trap $\mathrm{H} 2(\approx 600 \mathrm{meV})$ level can be identified.

the passivation of defects in the PDT samples and could be one reason for the improvement in $V_{\mathrm{OC}}$. Nevertheless, effects like increased carrier concentration lead to the $V_{\mathrm{OC}}$ enhancement as well [11]. An estimation by using the ideal diode equation confirms this. Using

$$
\Delta V_{\mathrm{OC}}=\frac{k T}{q} \ln \left(\frac{N_{\mathrm{A}, \mathrm{wPDT}}}{N_{\mathrm{A}, \mathrm{w} / \mathrm{oPDT}}}\right),
$$

an increase of around $13-16 \mathrm{mV}$ can be calculated by assuming the carrier concentrations in Table 1 [12]. Therefore, the main part of the $V_{\mathrm{OC}}$ increase can be explained by the increase of the carrier concentration.

In addition, DLTS measurements with a short filling pulse of $1 \mathrm{~ms}$ are shown in Figure 2 as dashed lines. For both samples with and without PDT, the DLTS signal changes significantly. In both cases, the strong signal for high temperatures reduces strongly. Furthermore for the short pulse width, no extremum is observable for the untreated sample. In contrast, the sample with PDT reveals some new features: a small positive peak at $150 \mathrm{~K}$ and one peak at high temperatures around $300 \mathrm{~K}$ which is cut due to the limited temperature window. Due to their positive direction, the peaks represent majority trap signals and will be denoted as "H1" and "H2", respectively. The evaluation of the $150 \mathrm{~K}$ peak leads to a trap level of $200 \mathrm{meV}$ above the valence band edge with a trap density of $(1.2 \pm 0.3) \times 10^{13} \mathrm{~cm}^{-3}$. Due to its low energy level it can be assumed not to be an effective recombination center. The small value of approx. $1.4 \times 10^{-17} \mathrm{~cm}^{2}$ for the capture cross section may be a hint to support this statement. In this measurement it was not possible to extract trap parameters for the high temperature peak, caused by insufficient data quality in this temperature range. Nevertheless, an additional investigation of the high temperature signal is given in the next section. For the traps $\mathrm{E} 1$ and $\mathrm{H} 2$, the evaluation values from DLTFS analysis confirmed the manual analysis.

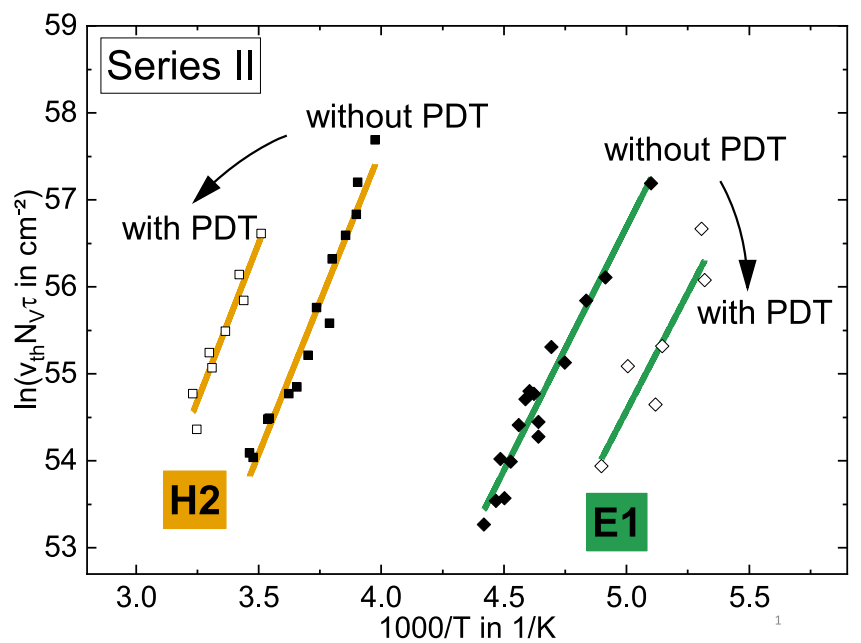

Fig. 4. Corresponding Arrhenius plot to the DLTS measurements of the Series II (compare Fig. 3).

The following section will describe measurements on samples of a second deposition series with and without PDT. As can be seen in Figure 3, a similar behaviour like in Series I can be recognized. In this figure, temperature scan measurements of samples with and without PDT are shown. Again the E1 signal at $220 \mathrm{~K}$ is observed and almost vanishes for the sample with PDT. Additionally, a prominent peak at $300 \mathrm{~K}$ was measured even for long filling pulses, corresponding to the $\mathrm{H} 2$ signal in Series I. For both peaks and both samples, a maximum analysis revealed the Arrhenius plot in Figure 4. The results of the linear fits are plotted in coloured lines. The data with yellow regression lines represent the evaluation of the $\mathrm{H} 2$ majority peak, the data with green regression lines the evaluation of the E1 minority peak. For the E1 signal, energy levels of $E_{\mathrm{T}, \mathrm{E} 1, \mathrm{w} / \mathrm{oPDT}}=(451 \pm 32) \mathrm{meV}$ and $E_{\mathrm{T}, \mathrm{E} 1, \mathrm{PDT}}=(459 \pm 11)$ meV are calculated. For the $\mathrm{H} 2$ signal $E_{\mathrm{T}, \mathrm{H} 2, \mathrm{w} / \mathrm{oPDT}}=$ $(608 \pm 8) \mathrm{meV}$ and $E_{\mathrm{T}, \mathrm{H} 2, \mathrm{PDT}}=(641 \pm 16) \mathrm{meV}$ are calculated. Within errors, they represent the same trap levels, respectively. Again, the manual evaluation values have been validated by the DLTFS evaluation. What should be noted here is that a superposition of the peaks can be observed. The fact that the two peaks (E1 and H2 signal) do not appear clearly separated leads to a possible source of error. However, in this study, the magnitude of this error cannot be considered. Since both traps, E1 and H2, have an energetic position near the middle of the CIGS band gap $\left(E_{\mathrm{g}, \mathrm{CIGS}}=1.15 \mathrm{eV}\right)$ with around $100 \mathrm{meV}$ difference and show reasonably high cross-sections and trap densities, both traps are likely candidates to be detrimental recombination centers influencing the device performance. All resulting trap properties are summarized in Table 1 in row "Series II". While the trap density of the E1 signal is reduced by a factor 3 (as seen comparably in Series I) from $(2.3 \pm 0.3) \times 10^{13} \mathrm{~cm}^{-3}$ to $(7.8 \pm 2.3) \times$ $10^{12} \mathrm{~cm}^{-3}$ due to the PDT, no significant change in $N_{\mathrm{T}}$ can be recognized for the $\mathrm{H} 2$ defect. The different evaluation methods result in ambiguous values for the trap density and in average similar densities are calculated. Hence, a 


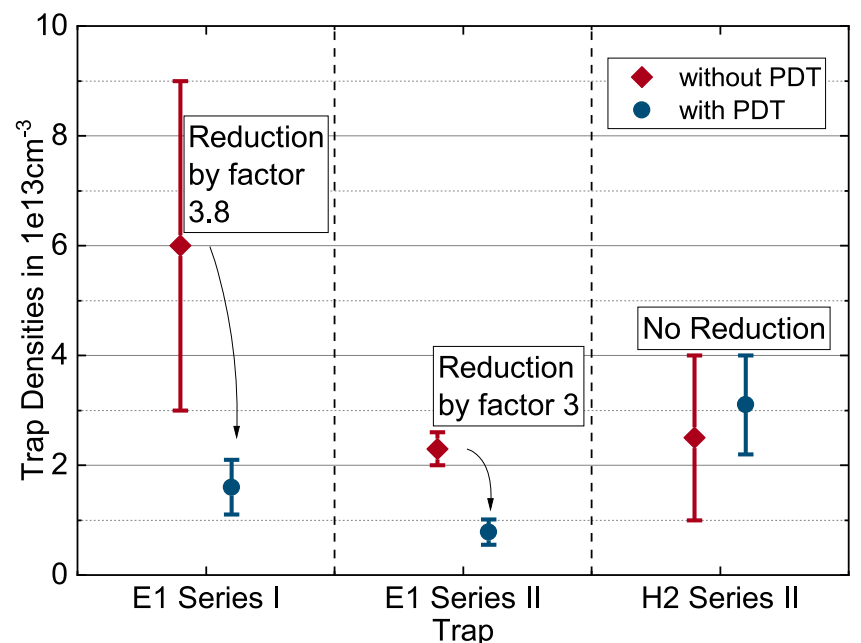

Fig. 5. Trap density data for $\mathrm{E} 1$ and $\mathrm{H} 2$ traps measured in this study.

significant reduction of trap density due to PDT cannot be observed. Figure 5 summarizes the results of the effect of PDT on the trap densities. For Series I densities of trap E1 are shown and for Series II the densities of traps E1 and H2.

Karki et al. reported similar results as they show DLTS measurements with a strong peak at room temperature, which they assign to an energy level of $570 \mathrm{meV}$ above $E_{\mathrm{V}}$, corresponding with the H2 signal in this study [11]. They investigated the effect of $\mathrm{RbF}$ as well, and observed no reduction of the density of this trap, supporting the present results. Deitz et al. show measurements of a $560 \mathrm{meV}$ trap in a silver alloy of CIGS $(\mathrm{Ag}, \mathrm{Cu})(\mathrm{In}, \mathrm{Ga}) \mathrm{Se}_{2}$ (ACIGS) as well and assigned it to the $\mathrm{Cu}_{\text {In/Ga }}$ substitutional defects as the most likely source [13]. Ab initio DFT calculations by Zhang et al. yield a trap energy of $580 \mathrm{meV}$ for $\mathrm{Cu}_{\text {In }}$ traps in $\mathrm{CuInSe}_{2}$ (CIS) which confirms this assignment even more. In the energy range of trap E1 they calculated a energy level at $410 \mathrm{meV}$ for indium vacancies $V_{\text {In }}$. Experimentally the minority trap E1 around $220 \mathrm{~K}$ was observed before by Kerr et al. in CIS [14]. They report an energy level of $520 \mathrm{meV}$ beyond the conduction band edge.

A possible explanation for the different behaviours of the $\mathrm{E} 1$ and $\mathrm{H} 2$ trap with the PDT is that RbF treatment only affects the E1 trap by passivation and in contrast the H2 trap is not affected. This could be linked with the spatial origin of the defects. From atom probe tomography (APT) measurements it is known that PDT has the effect that $\mathrm{Rb}$ [15] and other alkali [16] segregate at grain boundaries and interfaces. In this study, only a reduction of the trap density of $\mathrm{E} 1$ and not $\mathrm{H} 2$ is observed. Combining both facts leads to the conclusion that the E1 minority trap has its spatial origin at grain boundaries or interfaces. This agrees with the findings of Paul et al. [17]. They used nano-DLTS and located a trap with an energy level of $470 \mathrm{meV}$ above $E_{\mathrm{V}}$ (similar to our E1 level) at grain boundaries. Our study demonstrates that only this trap is passivated by the RbF$\mathrm{PDT}$. In contrast, the $\mathrm{H} 2$ trap is located in grain interior regions or is distributed more homogeneously through the layer. Alkali elements have a limited solubility in CIGS grain interiors [16] and therefore a significant passivation of the point defect H2 is not possible. Segregated at grain boundaries, the $\mathrm{Rb}$ atoms can occupy the defects and passivate them. However, further investigations are required to verify this theory and to distinguish between point and extended defects, for example through dependencies of the pulse width $[18,19]$.

\section{Conclusions}

In this work we analyzed the impact of RbF-PDT on the defect spectra of CIGS absorber layers from a productionlike co-evaporation inline system. The shown results are based on samples from two independent deposition series. In summary three different traps can be identified:

- Minority trap E1 at approx. $400 \mathrm{meV}$ : observed in almost every sample for long filling pulses. This trap shows a high sensitivity to RbF-PDT.

- Majority trap H1 at approx. $200 \mathrm{meV}$ : sometimes observed in samples with PDT, only for short filling pulses. Due to its low energy level and small capture cross-section, it is not a detrimental trap for the cell efficiency.

- Majority trap H2 at approx. $600 \mathrm{meV}$ : regularly observed for different measurement parameters, but sometimes difficult to evaluate. Similar results are reported in literature $[11,13]$.

Due to their energy levels and high trap densities of $\approx 1 \times 10^{13} \mathrm{~cm}^{-3}$, the traps $\mathrm{E} 1$ and $\mathrm{H} 2$ are both likely candidates to have an effect on the cell performance. A key result of this study is that a reduction of the trap density of the minority E1 trap can be observed by a factor of approximately 3 to 3.8 due to the alkali treatment. This confirms the passivating effect of rubidium in CIGS solar cells. In contrast, the majority trap $\mathrm{H} 2$ is not affected by the PDT process. The fact that only the E1 trap density is reduced due to PDT is explained with its origin at grain boundaries or interfaces in contrast to the $\mathrm{H} 2$ trap. Further investigations are required to strengthen this explanation. Despite this significant reduction of the E1 trap density, an effect on cell performance due to the reduced SRH recombination is not clearly determinable, because the main reason for the improvement can be assigned to the increase of the CIGS charge density.

However, these results serve as an important advancement in understanding defects in CIGS solar cells and the effect of PDT within the device. Since the shown studies were conducted on samples from a production-like inline system, these results are assumed to be transferable to absobers from large scale CIGS production.

\section{Supplementary material}

Figure S1: Information on carrier density calculations. Figure S2: Composition gradient and accessed region by DLTS pulse.

Figure S3: Pulse width scan.

Figure S4: Verification of evaluation results from Arrhenius plots. 
The Supplementary Material is available at https://www. epjpv.org/10.1051/epjpv/2022003/olm.

This work was financed by the German Federal Ministry for Economic Affairs and Energy (BMWi) under the contract number 0324353A (CIGSTheoMax) and support by the Ministry of Science, Research and the Arts of Baden-Württemberg as part of the sustainability financing of the projects of the Excellence Initiative II.

\section{Author contribution statement}

T.H. and A.K. performed the measurements and evaluations, A.B., T.M.F. and M.P. were involved in planning and supervised the work, S.P. manufactured the samples. T.H. processed the experimental data, performed the analysis and drafted the manuscript. M.Z. aided in interpreting the results and in designing the figures. All authors discussed the results and commented on the manuscript.

\section{References}

1. M. Nakamura, K. Yamaguchi, Y. Kimoto, Y. Yasaki, T. Kato, H. Sugimoto, IEEE J. Photovolt. 9, 1863 (2019)

2. D. Lang, J. Appl. Phys. 45, 3023 (1974)

3. A. Chirilă, P. Reinhard, F. Pianezzi, P. Bloesch, A.R. Uhl, C. Fella, L. Kranz, D. Keller, C. Gretener, H. Hagendorfer, et al., Nat. Mater. 12, 1107 (2013)

4. P. Jackson, R. Wuerz, D. Hariskos, E. Lotter, W. Witte, M. Powalla, Phys. Status Solidi Rapid Res. Lett. 10, 583 (2016)

5. S. Siebentritt, E. Avancini, M. Bär, J. Bombsch, E. Bourgeois, S. Buecheler, R. Carron, C. Castro, S. Duguay, R. Félix et al., Adv. Energy Mater. 10, 1903752 (2020)
6. N. Nicoara, R. Manaligod, P. Jackson, D. Hariskos, W. Witte, G. Sozzi, R. Menozzi, S. Sadewasser, Nat. Commun. 10, 1 (2019)

7. D. Hariskos, P. Jackson, W. Hempel, S. Paetel, S. Spiering, R. Menner, W. Wischmann, M. Powalla, IEEE J. Photovolt. 6, 1321 (2016)

8. A. Kanevce, S. Paetel, D. Hariskos, T.M. Friedlmeier, EPJ Photovoltaics 11, 8 (2020)

9. PhysTech, Hera-DLTS Software Manual (2013), www. phystech.de

10. S. Weiss, R. Kassing, Solid State Electron. 31, 1733 (1988)

11. S. Karki, P. Paul, G. Rajan, B. Belfore, D. Poudel, A. Rockett, E. Danilov, F. Castellano, A. Arehart, S. Marsillac, IEEE J. Photovolt. 9, 313 (2019)

12. R.A. Sinton, A. Cuevas, Appl. Phys. Lett. 69, 2510 (1996)

13. J.I. Deitz, P.K. Paul, R. Farshchi, D. Poplavskyy, J. Bailey, A.R. Arehart, D.W. McComb, T.J. Grassman, Adv. Energy Mater. 9, 1901612 (2019)

14. L.L. Kerr, S.S. Li, S.W. Johnston, T.J. Anderson, O.D. Crisalle, W.K. Kim, J. Abushama, R.N. Noufi, Solid State Electron. 48, 1579 (2004)

15. P. Schöppe, S. Schönherr, P. Jackson, R. Wuerz, W. Wisniewski, M. Ritzer, M. Zapf, A. Johannes, C.S. Schnohr, C. Ronning, ACS Appl. Mater. Interfaces 10, 40592 (2018)

16. H. Aboulfadl, K.V. Sopiha, J. Keller, J.K. Larsen, J.J. Scragg, C. Persson, M. Thuvander, M. Edoff, ACS Appl. Mater. Interfaces 13, 7188 (2021)

17. P.K. Paul, D.W. Cardwell, C.M. Jackson, K. Galiano, K. Aryal, J.P. Pelz, S. Marsillac, S.A. Ringel, T.J. Grassman, A.R. Arehart, IEEE J. Photovolt. 5, 1482 (2015)

18. A. Urbaniak, K. Macielak, N. Barreau, P. Szaniawski, M. Edoff, J. Phys. Chem. Solids 134, 58 (2019)

19. S. Heo, J. Chung, H.I. Lee, J. Lee, J.B. Park, E. Cho, K. Kim, S.H. Kim, G.S. Park, D. Lee et al., Sci. Rep. 6, 30554 (2016)

Cite this article as: Tim Helder, Ana Kanevce, Andreas Bauer, Mario Zinßer, Stefan Paetel, Theresa Magorian Friedlmeier, Michael Powalla, DLTS investigations on CIGS solar cells from an inline co-evaporation system with RbF post-deposition treatment, EPJ Photovoltaics 13, 7 (2022) 